\title{
LE RÔLE DU
}

\section{MANUEL DE}

\section{FLE DANS UNE}

\section{APPROCHE (PLURI) ACTIONNELLE}

Lara Neves SOARES

RÉSUMÉ : Dans cet article l'auteur s'interroge sur le caractère épistémologique de la didactique des langues étrangères et secondes (désormais DDL). Cette étude, commencée dans le cadre de son mémoire de Master, a permis de constater que ce domaine connaît un changement paradigmatique depuis quelques années. Depuis lors elle s'intéresse aux diverses instances de validation des pratiques didactiques (méthodologues, didacticiens, marché éditorial, enseignants et apprenants) qui doivent rassembler les notions-clés du domaine autour d'un modèle vraisemblable. A cet effet, son texte a d'abord cherché à éclairer les dernières discussions en DDL à un niveau didactologique. Autrement dit, il y a eu une volonté d'établir un dialogue entre les notions trouvées dans notre domaine et leur possible applicabilité dans les pratiques en salle de classe à partir de l'exemple d'un manuel de FLE qui tient une place centrale dans l'approche ici nommée (pluri)actionnelle.

MOTS-CLÉS : didactologie, cohérence épistémologique, FLE, manuel, compétence.
1 Lara Neves Soares est doctorante à l'Université Paris Descartes - Sorbonne Paris Cité en Sciences du Langage et Didactique des Langues Étrangères. Sa recherche, depuis son Master, porte sur le caractère épistémologique de la didactique des langues étrangères, notamment du FLE, depuis l'arrivée du Cadre Européen Commun de Référence pour les Langues. Elle enseigne également le français et le portugais en tant que langues étrangères, en France. 
RESUMO: Neste artigo a autora questiona o caráter epistemológico da didática das línguas estrangeiras e segundas (a partir de agora DDL). O presente estudo, começado no contexto de sua dissertação de mestrado, permitiu constatar que essa área passa por uma mudança paradigmática há alguns anos. Desde então se interessa às várias esferas de validação das práticas didáticas (metodologistas, didáticos, mercado editorial, professores e aprendizes) que devem regrupar as noções-chave da área em torno de um modelo verossímil. Para isto, o texto buscou, num primeiro momento, esclarecer as últimas discussões em DDL a um nível didactolólogico. Em outras palavras, houve a necessidade de estabelecer um diálogo entre as noções encontradas em nossa área e a possível aplicabilidade delas nas práticas da sala de aula a partir do exemplo de um manual de FLE que tem um lugar central na abordagem aqui denominada (pluri)actional.

PALAVRAS-CHAVE: didactologia, coerência epistemológica, FLE, competência. 
La présente étude s'intéresse à l'approche méthodologique préconisée par le Conseil de l'Europe : la perspective actionnelle (dorénavant $\mathrm{PA}$ ) dans le Cadre Européen Commun de Référence pour les Langues (CECRL, CECR ou Cadre). L'intérêt pour un tel sujet part du constat suivant : en didactique des langues étrangères et secondes (DDL) le rôle tenu par les méthodologies d'enseignement-apprentissage est central et joue deux fonctions de cohérence : celle des pratiques en salle de classe et celle de l'épistémologie du domaine. Il nous paraît important d'insister sur cette notion de cohérence comprise comme cadre organisateur d'une discipline, encore jeune comparée à d'autres domaines, mais qui pour autant n'est pas plus simple ${ }^{2}$.

Il y a donc le besoin d'inscrire cette étude dans une voie tant épistémologique que synthétique. Ces notions sont empruntées à Demazière et Narcy-Combes (2007, p. 14) pour qui « la recherche épistémologique travaille à partir des publications scientifiques de la didactique et de ses domaines de référence. [...] Cette recherche [de synthèse] propose un état des lieux sur un point ou un concept du domaine », car nous estimons que les débats autour de ces problématiques sont loin d'être clos en DDL.

En outre, par rapport aux maintes discussions sur le Cadre, une, en spécifique, a attiré notre attention. C'est celle de Maurer (2011, p. 15) qui affirme que

La principale limite [du CECRL] est méthodologique. Les descripteurs fournis par compétence et par niveau sont certes utiles en tant que points de référence, que bornes sur un chemin à parcourir, mais ils ne disent rien des enseignements que l'on doit mener pour les atteindre, des contenus linguistiques détaillés ni, a fortiori, des progressions, un mot qui est peu utilisée du reste.

Cette observation est complémentaire à notre avis. Nous pensons que le flou méthodologique en didactique des langues qui règne de nos jours, en salles de classe, est un reflet de l'éclectisme méthodologique $^{3}$ dans le domaine.

Cette réflexion part, initialement, de l'analyse d'un manuel de $F^{4}{ }^{4}$ - étude effectuée dans le cadre de notre mémoire (cf. NEVES SOARES, 2012) - pour proposer une démarche de cohérence épistémologique en rapport à la lignée de pensée préconisée par le Conseil de l'Europe. Le manuel de langue est perçu alors comme un outil important qui joue, à divers degrés, un rôle primordial dans le travail de l'enseignant et de l'apprenant. Il peut également être un élément for-
2 La simplicité a difficilement une place en DDL qui, depuis son apparition à la fin du XVIIIe siècle, se présente comme un carrefour de savoirs venus de différents domaines : la linguistique, la pédagogie, la psychologie, la sociologie, l'ethnologie entre autres. La notion de cohérence est, par conséquent, fortement liée à l'idée de complexité qui, elle-même, convoque celle de structuration et par tant, la notion de progression qui importe dans ce cadre.

3 Nous reprenons le terme crée par Puren, car il a été utilisé dans le contexte des méthodologies d'enseignementapprentissage, celui qui est le notre aussi. Cf. PUREN, C. La didactique des langues à la croisée des méthodes. Essai sur l'éclectisme. Paris : CREDIF, Didier, 1994, p. 7-59.

4 LABASCOULE, J. et al. Nouveau Rond-point pas à pas A1. Livre de l'élève. Paris : Editions Maison des langues, 2010. 
mateur de l'enseignant (cf. VERDELHAN-BOURGADE, 2004) qui, lors du choix du manuel (et de l'imposition dans certains cas), se retrouve confronté à une certaine méthodologie de travail à mettre en œuvre. Les manuels évoluent et s'adaptent selon les méthodologies en vogue, le contexte institutionnel présenté et les méthodes pratiquées, et « représentent en effet, dans la pratique quotidienne de l'enseignant, une référence à la fois permanente et concrète à la volonté, parfois changeante et toujours abstraite, de l'institution. »(CHOPIN, 2000, p. 82).

C'est donc en partant du principe que les manuels de FLE suivent le courant méthodologique d'une époque et que leur utilisation en salle de classe relève des pratiques et des stratégies à mettre en œuvre, que nous proposons cette réflexion entre la cohérence de la méthodologie et des méthodes proposées par ce manuel avec la(les) méthodologie(s) en vogue aujourd'hui. Et aussi, parce que, depuis quelques années, dans le domaine de la DDL, on constate un affaiblissement de la discussion du comment faire, comme le signale Beacco (2007, p. 15) : «Les méthodologies d'enseignement des langues (couramment dites méthodologies tout court, dans le métier) ne sont plus, comme il y a quelques années, au centre de la réflexion en didactique des langues. Les publications qui leur sont consacrées sont désormais peu nombreuses, pour ne pas dire rares ». Bien que les recherches soient tournées vers le rôle de l'apprenant en tant qu'acteur de son discours et de son apprentissage, cela ne veut pas dire que la question méthodologique ne reste pas capitale lors de la réalisation d'un cours.

Pour ce travail nous partons de quelques hypothèses concernant l'étude déjà menée avec Le Nouveau Rond Point A1 (manuel de FLE) et l'objectif de cette analyse (la cohérence théorique de la perspective actionnelle) en tenant compte des notions-clés utilisées dans le domaine.

Tout d'abord, malgré les dernières recherches sur la transposition didactique des manuels à l'ère de l'actionnel (cf. BENTO 2013, 2014 ; RIQUOIS, 2014), les discussions autour du comment faire dans la perspective actionnelle, surtout avec cet outil, ne sont pas nombreuses. D’après notre expérience et notre observation sur le terrain, nous avons pu observer que, dans le corps enseignant, il existe une réelle difficulté à mettre en œuvre l'approche préconisée par le Conseil de l'Europe. Un des motifs est que « l'apprentissage est ainsi [dans l'approche actionnelle] plus fortement contextualisé, mis en rapport avec la situation dans laquelle il s'inscrit, et le lecteur est invité à oublier tout enseignement trop formaté pour s'orienter vers une adaptation complète à la situation locale. » (RIQUOIS, 2014, p. 94). Situation ac- 
centuée par la mise en place d'un travail qui préconise aussi un acteur social alors que précédemment on parlait d'apprenant (cf. ROSEN, 2009). Cela veut dire que l'apprentissage doit porter sur la notion de sujet participant et créateur de son environnement avec d'autres personnes ou en groupe. Il ne s'agit plus simplement des inter-actions, mais aussi des «co-actions » (terme utilisé d'abord par Puren, 2002), ce qui agrandit et complexifie les appareils pédagogiques. Ce changement est plus que simplement nominatif. En changeant les préfixes, ce sont de nouvelles définitions qu'il faut revoir. Nous rejoignons Puren (PUREN, 2002, p. 6) quand il explique pourquoi l'utilisation du terme « co-action » : « il [ce terme] marque clairement l'évolution de la perspective actionnelle entre l'AC [l'approche communicative] et la PA [la perspective actionnelle], ou l'on passe de l' interaction à la co-action, [...] il me permet de proposer parallèlement le concept de co-culturel en opposition a celui d' interculturel » Cette pensée est complémentaire à notre proposition de démarche dans cet article.

Ensuite, il est important de questionner le caractère officiel et unificateur du CECRL dans l'enseignement-apprentissage des langues au niveau des contextes non-européens. Or, si les manuels sont conçus et basés selon les descripteurs du CECRL (rappelons qu'il s'agit d'un Cadre de Référence Européen), il est clair que l'appropriation de tel outil peut s'avérer difficile, voire décontextualisée selon les différentes cultures éducatives. Le mot contexte, comme nous venons de le voir chez Puren nous apparaît dans la PA comme fil conducteur des démarches à suivre. C'est pourquoi, le manuel gagne un poids si central en classe de langue, vu qu' " il est ainsi une illustration du processus de transposition didactique, intermédiaire entre les programmes prescrits, les savoirs savants et les pratiques professionnelles (Perrenoud, 1984 ; Bruillard, 2005 : 22) » comme nous souvient Bento (2014, p. 106).

Enfin, la notion de compétence attire notre attention sur le travail échelonné proposé par le CECRL et avec elle l'idée de progression linguistique en cours de langue. Richer (2012, p. 56) présente bien la situation crée par l'arrivée du Cadre quand il affirme que

Ce modèle de la compétence à communiquer langagièrement, preuve d'une accentuation de la démarche taxinomique d'énumération de compétences déjà à l'œuvre dans le Communicatif, se présente sous la forme de compétence en cascades, [...], ce qui ne peut qu'entraîner un effet de brouillage suscité par la désignation, sous le même terme de compétence, de niveau de conceptualisation différente. 
Suite aux points présentés, il nous a semblé pertinent, après l'étude menée sur un manuel de FLE (cf. NEVES SOARES, 2012), de revenir sur les points théoriques de la perspective actionnelle. Les discussions sur des propositions qui relèvent de la pratique en salle de classe peuvent servir d'aide auprès des enseignants qui ne sont, peutêtre, pas familiarisés (voire à l'aise) avec la PA.

\section{La méthodologie}

À partir d'une revue de la littérature sur la cohérence méthodologique de la perspective actionnelle et suite aux résultats déjà obtenus dans le cadre du mémoire de master, nous proposons de traiter la question de la mise en place de la PA en salle de classe en la considérant une méthodologie à part entière en DDL. Pour ce faire, prenons le manuel de FLE comme l'outil pédagogique essentiel tenant rôle de médiateur dans la transposition didactique entre le CECRL et les enseignants de FLE. Dans cette même lignée, Riquois (2014) a observé que les termes didactiques utilisés par les manuels de FLE n'étaient pas clairs quant à leur acception. L'auteur affirme que « cette imprécision terminologique est sans aucun doute le symptôme d'une imprécision théorique qui empêche toute idée de transposition du texte de référence à la pratique de la classe par le biais du manuel. » (RIQUOIS, 2014, p. 103). Ce qui nous fait insister sur la notion de cohérence épistémologique dans la DDL à travers les méthodologies d'enseignement-apprentissage mises en pratique.

\section{Un axe de travail possible}

Ainsi, nous proposons une démarche que nous nommons (pluri)actionnelle. Cette démarche se construirait selon les besoins des acteurs et chaque classe travaillerait et progresserait de façon unique. Il est clair que « la perspective actionnelle amène l'enseignant à développer ses compétences de concepteur de matériel pédagogique. Il ne se limite plus à créer des activités qui vont se juxtaposer, il doit désormais concevoir un ensemble prenant place dans un contexte plus précis. » (DURIETZ, JÉRÔME, 2009, p. 66) Toutefois, c'est aussi par le biais de l'action que l'apprenant pourra assumer le rôle d'acteur social responsable de son apprentissage et dans ce sens « alléger » le travail de l'enseignant.

En effet, les sujets impliqués dans le processus d'enseignement-apprentissage des LE sont aussi, en quelque sorte, les agents 
d'une telle situation, c'est-à-dire que les conduites dans notre domaine sont complexes parce que l'objet (l'enseignement-apprentissage d'une langue) et les sujets (les enseignants, les apprenants, les méthodologues, les didactologues etc.) le sont également. Le risque, à notre avis, est que ce discours d'éclectisme et complexité laisse place au relativisme en mettant le domaine de la DDL dans un état de résignation au nom de la diversité.

La diversité doit être source de propositions et de recherches qui visent à centrer les discussions afin de favoriser la mise en pratique des concepts didactiques. Cette même diversité ne doit pas être vue comme nuisible, mais plutôt comme un axe de travail. Si un changement de paradigme semble s'opérer dans le domaine, il n'est pas anodin. Mais si avec ce changement le discours relativiste se systématise, nous devons être sur nos gardes. Ce changement met l'action au centre des intérêts épistémologiques, comme nous le rappelle Chini (2010, p. 164) :

On est passé d'un intérêt de type pragmatique pour les actes de parole dans un cadre conversationnel à une logique de l'action sociale, appréhendée comme unité dynamique, orientée vers un but concret et qui, si elle intègre une dimension langagière, conduit à considérer le rôle du langage non plus comme le vecteur fondamental mais comme un moyen parmi d'autres au service de cette action.

Dans la PA, il ne s'agit donc plus d'inter-agir avec l'autre, mais plutôt de co-agir ; d'être capable de prendre en compte la dimension sociale du langage et les éléments du contexte où il est présent. Cette dimension de groupe est construite parallèlement à celle d'individu. L'un ne peut exister sans l'autre. Dans les nombreuses activités proposées par le Nouveau Rond-Point, avons pu constater que l'interaction (fort présente depuis l'AC) est une démarche très utilisée par les auteurs dans la tentative de mettre les apprenants dans une utilisation authentique de la langue, mesurée selon leurs besoins (mais insuffisantes, car le motif qui était au départ de ces échanges avait encore un caractère très peu actionnel). Cela veut dire qu'on était dans la majorité des cas dans l'optique d'apprendre une langue pour communiquer et non en tant que communication. La conclusion de Bento (2014) dans une récente étude vient soutenir notre observation : « tous les auteurs des manuels proposent des activités en interaction, mais s'expriment finalement très peu sur leurs intérêts. » (BENTO, 2014, p. 111).

Notre démarche (pluri)actionnelle propose donc de placer ces 
interactions en tant que méthode de la mise en place de l'action. À notre avis, de cette façon, nous pourrons envisager un changement paradigmatique entre l'AC et la $\mathrm{PA}$ - changement qui est à construire (cf. RICHER, 2014) ${ }^{5}$, puisqu'ainsi on rendrait compte du système complexe que les participants des échanges devraient mobiliser afin de maintenir ce contact (langagier ou non). Les interactions dans ce sens se font entre "individu - milieu », mais surtout entre "individu groupe ». C'est pourquoi l'acquisition des compétences générales individuelles et des stratégies d'apprentissage - telles qu'elles sont présentées dans le CECRL - sont essentielles pour la composition et la mobilisation de la compétence communicative.

Avec la PA, nous dépassons le principe de la communication comme caractéristique centrale de l'enseignement-apprentissage des LE pour aboutir à l'action comme élément déclencheur et aussi final de toute interaction (voire communication) sociale. En effet c'est seulement inséré dans un contexte et une situation que l'individu prend son rôle, c'est-à-dire qu' "il faut concevoir la personne non comme un agent isolé, mais comme un co-agent immergé dans une situation historiquement déterminée qui compose un champ de transactions où, avec d'autres agents, il instaure un rapport aux mondes qu'ils construisent ensemble. »(VERNANT, 1997, p. 151).

Dans cette optique, il fallait donc proposer, dans les manuels des LE, des tâches qui prennent en compte cette complexité des échanges humains, où les compétences sociolinguistiques guideraient la progression du schéma de classe tout en s'appuyant sur le caractère actionnel du langage pour conduire au discours. Nous sommes bien d'accord qu'une telle approche relève du savoir-faire de tous les sujets participants et qu'un travail dans une perspective actionnelle demande aussi un changement dans les différentes pratiques éducatives des différentes cultures (voire dans la formation continue des enseignants).

La démarche (pluri)actionnelle repose ainsi sur certaines compétences selon le contexte où elle sera pratiquée. Ce qu'il faut remarquer dans ce type de travail c'est qu'une fois dans la vraie action, l'interaction n'est pas prévisible. Certes, on peut prévoir les échantillons de LE nécessaires mais pour avoir un certain contrôle de la progression linguistique il faudra revenir aux activités pédagogiques, car, dans ce cas, « la langue prime sur le discours, l'instance communicationnelle semble être stopée au profit de la focalisation sur le code. » (CICUREL, 2011, p. 61). L'important ici c'est de mettre les apprenants dans des actions qui les amènent à être des vrais acteurs sociaux en salle de
5 Ce nouveau paradigme méthodologique de la perspective actionnelle, parce qu'il accorde une égale importance tant aux composantes psycho-socio-cognitives et culturelles qu'aux composantes linguistiques et sociolinguistiques, constitue une approche plus holistique de l'enseignement / apprentissage des langues. Mais il est encore largement à l'état programmatique. Aussi nécessite-t-il, afin de l'asseoir plus solidement, de lancer de nombreux travaux de recherche. » (RICHER, 2014, p. 45). 
classe et ainsi pouvoir trouver des pistes sur les différentes méthodes qui peuvent constituer la perspective actionnelle.

En considérant la PA comme une méthodologie à part entière, il nous reste l'absence de positionnement théorique pour la part du CECRL à prendre en compte. C'est en ce moment que le manuel de langue assume un rôle central. Si le Cadre s'abstient de toute responsabilité d'imposition méthodologique, la logique du marché éditorial se positionne différemment. Comme le constate Bourguignon (2010, p. 10): « [...] on peut se demander pourquoi faire tant de bruit autour du CECRL s'il n'y a presque rien qui change ! Il est vrai que ce 'presque rien qui change' se reflète dans les manuels scolaires. » Des manuels se disant actionnels, nous en avons des centaines et c'est l'appropriation de ces outils pédagogiques qui peut générer des doutes.

Pour notre démarche, il faut prévoir un travail en salle de classe qui repose sur la compétence sociolinguistique de l'apprenant comme point de départ pour revenir à la complexité du langage et de la communication dans les échanges humains et dans les rapports du groupe-classe, car c'est la culture qui guidera la progression de l'action et de la langue. De Carlo (1998, p. 97) nous éclaire sur ce sujet en déclarant que :

Pour revenir à notre modèle de parcours en spirale - partir du connu, de l'évident, du 'naturel', de l'universel, pour entrer en contact avec l'inconnu, l'étonnant, l'étrange, le relatif qui déviant, à son tour, le point de départ d'une réinterprétation de soi -, cette phase de réflexion sur soimême devrait avoir préparé le terrain pour une ouverture vers les autres.

C'est justement cette « ouverture vers les autres » qu'il faut chercher dans la co-action. Il est important de préciser que les doutes autour d'une démarche actionnelle semblent venir du contexte dans lequel effectuer la communication, parce que si le CECRL (2001, p. 41) précise que :

Il est depuis longtemps admis que l'usage de la langue varie très largement selon les exigences du contexte dans lequel elle apparaît. De ce point de vue, la langue n'est pas une expression neutre de la pensée comme peuvent l'être, par exemple, les mathématiques. Le besoin et le désir de communiquer naissent d'une situation donnée et la forme comme le contenu de la communication répondent à cette situation. 
En effet, le contexte joue un rôle important dans l'enseignement-apprentissage de la LE (cf. BEACCO, CHISS, CICUREL, VÉRONIQUE, 2005). Dans un premier moment les interactions se développent dans un domaine éducationnel, mais parfois les apprenants qui y sont insérés n'ont pas besoin d'apprendre la langue pour communiquer dans ce domaine. Ils doivent l'apprendre pour agir dans un autre secteur de leur vie sociale (personnel, professionnel, public etc.). Mais si l'on veut faire de l'apprenant un « acteur social à part entière » (PUREN, 2009, p. 123), le manuel devra proposer la langue dans sa « double nature », c'est-à-dire « objet d'étude et outil de communication »(BOURGUIGNON, 2010, p. 53).

La notion de centration s'applique fortement dans cette démarche, mais elle est placée selon les objectifs visés. Cela veut dire que l'apprenant assume le discours de l'utilisateur social de la LE et l'enseignant s'occupe de la progression du discours. A ce propos, Chiss (2000, p. 70) déclare que :

[...] s'il fallait absolument 'centrer', alors [...] je centrerai sur 'le savoir de l'enseignant', 'le savoir de l'enseignant' dans la mesure où nous avons affaire à des processus sociaux d'appropriation des connaissances, et donc à la nécessité d'avoir déjà construit pour construire avec les élèves.

\section{Conclusion}

Le manuel, dans notre démarche, est l'outil pédagogique essentiel pour offrir ce dialogue entre enseignant - apprenant ; enseignant - langue-culture ; langue-culture - apprenant et enfin acteurs sociaux - discours.

Insister sur les compétences sociolinguistiques au sommet du processus signifie donner du sens à la notion d'acteur social. C'est pourquoi les cultures éducatives ont une position assez importante dans les discussions en didactique actuellement. Nous ne pouvons pas les effacer ou les nier parce qu'elles donnent justement le caractère multiculturel et multilingue du continent européen et des échanges internationaux. Cependant, cette diversité des contextes ne doit pas donner lieu au flou méthodologique, au contraire, elle doit être source de ressources dans une démarche (pluri)actionnelle. C'est la co-action qui guide les pratiques et c'est aussi par la co-action (plurielle) que la langue-culture est acquise. Il reste à savoir si la mise en place de telle proposition déboucherait sur les mêmes résultats. 
LABASCOULE, J. \& A1. Nonvean Rond-point pas à pas A1. Livre de l'élève. Paris : Editions Maison, 2010.

\section{RÉFÉRENCES BIBLIOGRAPHIQUES}

BEACCO, J-C. L'approche par compétences dans l'enseignement des langues. Paris : Didier, 2007.

BEACCO J-C., CHISS J-L., CICUREL F., VÉRONIQUE D., eds. Les cultures éducatives et linguistiques dans l'enseignement des langues. Paris : PUF, 2005.

BENTO, M. La perspective actionnelle dans les manuels de langue au collège. Recherches en didactiques. Cahiers Théodile, n 15. 2013.

BENTO, M. "Les auteurs de manuels : transpositeurs à l'interface entre savoirs savants et savoir à enseigner ». In: Recherches et applications, $\mathrm{n}^{\circ}$ 55, « La transposition en didactique du FLE et du FLS », FIPF, Clé International, p. 106 - 119, janvier 2014.

BERTOCCHINI, P. COSTANZO, E. Manuel de formation pratique pour le professeur de FLE. Paris, CLE International, 2008.

BOURGUIGNON, C. Pour enseigner les langues avec le CECRL - clés et conseils. Paris : Delagrave, 2010.

CHINI, D. «Approche actionnelle, plurilinguisme et conceptualisation linguistique ». In : CHINI, D., GOUTÉRAUX, P. éd., Psycholinguistique et didactique des langues étrangères. Paris : Ophrys, p. 5-18, 2008.

CHINI, D. « Langage et/ou action ? La perspective actionnelle favorise-t-elle vraiment l'apprentissage linguistique ? »In : Recherches et applications, $\mathrm{n}^{\circ} 48$, "Interrogations épistémologiques en didactique des langues, Clé International, p. 164 - 175, juillet 2010.

CHISS, J-L. « La progression : un problème typiquement didactique. » In : COSTE, D. dir. La Notion de Progression, $\mathrm{n}^{\circ} 3$ mars, p. 67-70 Fontenay/Saint Cloud : ENS Éditions, 2000.

CHOPIN, A. « Le rôle des manuels. » In : COSTE, D. dir. La Notion de Progression, $n^{\circ} 3$ mars, p. 81-85, Fontenay/Saint Cloud : ENS Éditions, 2000. 
CICUREL, F. Les interactions dans l'enseignement des langues. Agir professoral et pratiques de classe. Paris : Didier, 2011.

CONSEIL DE L'EUROPE. Cadre européen commun de référence pour les langues. Paris : Didier, 2001.

DE CARLO, M. L'interculturel. Paris, CLE International, 1998.

DEMAZIERE, F., NARCY-COMBES, J-P. Du positionnement épistémologique aux données de terrain. Les Cahiers de l'Acedle, numéro 4, juin 2007.

DURIETZ, S., JÉRÔME, N. «Perspective actionnelle et approche basée sur scénario. Un compte rendu d'expérience aux Nations Unies ». In : ROSEN, E. Coord. La perspective actionnelle et l'approche par tâches en classe de langue. Le français dans le monde. Recherches et applications, $n^{\circ} 45$. Paris : Clé International, 2009.

MAURER, B. Enseignement des langues et construction européenne. Le plurilinguisme, nouvelle idéologie dominante. Paris : Éditions de Archives Contemporaines, 2011.

NEVES SOARES, L. La place de la complexité didactique dans le discours des acteurs sociaux. In: FRANCONTRASTE: L’AFFECTIVITÉ ET LA SUBJECTIVITÉ DANS LE LANGAGE. 2014. Anais do colóquio internacional da Universidade do Zagreb. CIPA : Mons. p. 333-337.

NEVES SOARES, L. La cohérence interne de la perspective actionnelle et le manuel de FLE comme outil pédagogique majeur. 2012. 91. Dissertação de mestrado - Université de Strasbourg, França.

PUREN, C. La didactique des langues à la croisée des méthodes. Essai sur l'éclectisme. Paris : CRÉDIF, Didier, 1994.

PUREN, C. «Perspectives actionnelles et perspectives culturelles en didactique des langues-cultures : vers une perspective co-actionnelle co-culturelle ». Les langues modernes, $n^{\circ} 3 / 2002$, p. 55-71.

PUREN, C. 2009. « La nouvelle perspective actionnelle et ses implications sur la conception des manuels de langue ». In :L'approche actionnelle dans l'enseignement des langues. Barcelone : Maison des langues, pp. 119-137, 2009.

RICHER, J-J. La didactique des langues interrogée par les compétences. Bruxelles : E.M.E, 2012.

RICHER, J-J. Conditions d'une mise en oeuvre de la perspective actionnelle en didactique des langues. Recherche et pratiques pédago- 
giques en langues de spécialité - Cahiers de l'APLIUT Volume XXXIII N ${ }^{\circ}$ 1, février 2014.

RIQUOIS, E. «Transposer le CECR dans les manuels de français langue étrangère : une gageüre ? ». In: Recherches et applications, $\mathrm{n}^{\circ} 55$, « La transposition en didactique du FLE et du FLS », FIPF, Clé International, p. 92 - 105, janvier 2014.

ROSEN, E. «Perspective actionnelle et approche par les tâches en classe de langue. » In : ROSEN, E. Coord. La perspective actionnelle et l'approche par les tâches en classe de langue. Le français dans le monde. Recherches et applications, n45. Paris : CLE International, 2009.

VERDELHAN-BOUGARDE, M. Le manuel en situation FLS pluri-culturelle : un rôle complexe. Marges linguistiques. Juillet, 2004.

VERNANT, D. Discours à l'action.Paris : Presses Universitaires de France, 1997. 